\title{
Light-front quark model consistent with Drell-Yan-West duality and quark counting rules
}

\author{
Thomas Gutsche ${ }^{1}$, Valery E. Lyubovitskij ${ }^{1}{ }^{*}$, Ivan Schmidt ${ }^{2}$, Alfredo Vega ${ }^{3}$ \\ 1 Institut für Theoretische Physik, Universität Tübingen, \\ Kepler Center for Astro and Particle Physics, \\ Auf der Morgenstelle 14, D-72076 Tübingen, Germany \\ 2 Departamento de Física y Centro Científico Tecnológico de Valparaíso (CCTVal), \\ Universidad Técnica Federico Santa María, \\ Casilla 110-V, Valparaíso, Chile \\ 3 Departamento de Física y Astronomía, \\ Universidad de Valparaíso, \\ Avenida Gran Bretaña 1111, Valparaíso, Chile
}

(Dated: August 3, 2018)

\begin{abstract}
We propose a phenomenological light-front wave function for hadrons with arbitrary twistdimension (mesons, baryons and multiquark states), which gives the correct scaling behavior of structure functions (parton distributions) and form factors for pions and nucleons. For other hadronic states the proposed wave function produces form factors consistent with quark counting rules, and gives predictions for structure functions (parton distributions). As an application we build a light-front quark model for nucleons based on the proposed wave function.
\end{abstract}

PACS numbers: 12.38.Aw, 12.38.Lg. 13.40.Gp, 14.20.Dh

Keywords: quark counting rules, nucleons, form factors, parton distributions

\section{INTRODUCTION}

The main objective of this paper is to propose a phenomenological light-front wave function (LFWF) for hadrons with arbitrary twist-dimension (mesons, baryons and multiquark states) which gives the correct scaling behavior of structure functions (parton distributions) and form factors both for pions and nucleons. For other hadronic states the proposed wave function produces form factors consistent with quark counting rules, and also gives predictions for the corresponding structure functions (parton distributions). As an application we construct a light-front quark model for nucleons based on the proposed wave function. This model is by construction consistent with the Drell-Yan-West (DYW) relation [1] between the large- $Q^{2}$ behavior of nucleon electromagnetic form factors and the large- $x$ behavior of the structure functions (see also Ref. [2] for the extension to inelastic scattering), and with quark counting rules [3]. Based on the findings of Refs. [1 13$]$ one can e.g. relate the behavior of the quark distribution function in the nucleon $q_{v}(x) \sim(1-x)^{p}$ at $x \rightarrow 1$ to the scaling of the proton Dirac form factor $F_{1}^{p}\left(Q^{2}\right) \sim 1 /\left(Q^{2}\right)^{(p+1) / 2}$ at large $Q^{2}$ (the parameter $p$ is related to the number of valence constituents $N$ in the hadron, hence for $N=3$ we have $p=3$ ). In Refs. [4, 5] the large- $x$ scaling of pion

\footnotetext{
*On leave of absence from Department of Physics, Tomsk State
} University, 634050 Tomsk, Russia and nucleon PDFs and GPDs has been obtained in the framework of perturbative QCD. In particular, the pion structure function behaves as $(1-x)^{2}$ at $x \rightarrow 1$, while nucleon spin non-flip and spin-flip structure functions behave at large $x$ as $(1-x)^{3}$ and $(1-x)^{5}$, respectively. The importance of these scaling laws and their role in the description of hadron structure has been stressed and studied in detail in the literature, see e.g. Refs. [4]- 7].

In this paper we show that these important features of nucleon structure can be also fulfilled in the framework of a simple light-front quark model based on a phenomenological wave function with a specific dependence on the transverse momentum $\mathbf{k}_{\perp}$ and the light-cone variable $x$. We also derive the phenomenological LFWF for hadrons with arbitrary twist-dimension $\tau$, which gives the required scaling for the corresponding form factors and provides predictions for unknown structure functions and GPDs.

\section{LIGHT-FRONT WAVE FUNCTION FOR HADRON WITH ARBITRARY TWIST}

In this section we propose a phenomenological LFWF for hadrons with arbitrary twist-dimension $\tau$ without referring to the flavor structure. We choose the form

$$
\begin{aligned}
\psi_{\tau}\left(x, \mathbf{k}_{\perp}\right) & =N_{\tau} \sqrt{\log (1 / x)}(1-x)^{\frac{\tau}{2}+\frac{\alpha(\tau)}{2}-1} \\
& \times \exp \left[-\frac{\mathbf{k}_{\perp}^{2}}{2 \kappa^{2}} \frac{\log (1 / x)}{(1-x)^{2-\alpha(\tau)}}\right]
\end{aligned}
$$


where

$$
N_{\tau}=\frac{4 \pi}{\kappa} \sqrt{1+\tau}
$$

is the normalization constant,

$$
\alpha(\tau)=\frac{2}{\tau-1},
$$

$x$ is the Bjorken variable, $\mathbf{k}_{\perp}$ is the transverse momentum and $\kappa$ is the scale parameter. Such type of LFWF is motivated by the soft-wall AdS/QCD model (see e.g. Ref. [8-13]) and corresponds to the dressed function of a hadron dual to the corresponding bulk profile confined in the quadratic dilaton potential. The idea to extract LFWFs by matching to AdS/QCD has originally been suggested in Ref. [8], considering the pion electromagnetic form factor in two approaches - AdS/QCD and light-front QCD. In a series of papers $8-13$ ] this problem was further discussed in detail. The LFWF derived in the present paper is different from the LFWFs directly extracted from AdS/QCD. The difference lies in an additional specific dependence on the light-cone variable, which is required to get consistency with modelindependent scaling rules. In particular, the effective LFWF extracted from AdS/QCD in Ref. [12], in the case of two-parton (meson) states, has the following form

$$
\psi_{\tau=2}\left(x, \mathbf{k}_{\perp}\right) \sim \frac{\sqrt{\log (1 / x)}}{1-x} \exp \left[-\frac{\mathbf{k}_{\perp}^{2}}{2 \kappa^{2}} \frac{\log (1 / x)}{(1-x)^{2}}\right] .
$$

The LFWF as proposed in (1) contains the extra factor

$$
f_{L}(x)=(1-x)^{2},
$$

depending on the light-cone variable with the result

$$
\begin{aligned}
\psi_{\tau=2}\left(x, \mathbf{k}_{\perp}\right) & \sim \frac{\sqrt{\log (1 / x)}}{1-x} f_{L}(x) \\
& \times \exp \left[-\frac{\mathbf{k}_{\perp}^{2}}{2 \kappa^{2}} \frac{\log (1 / x)}{(1-x)^{2}} f_{L}(x)\right] .
\end{aligned}
$$

The LFWF suggested here leads to the structure function (parton distribution) $\rho_{\tau}(x)$

$$
\rho_{\tau}(x)=\int \frac{d^{2} \mathbf{k}_{\perp}}{16 \pi^{3}}\left|\psi_{\tau}\left(x, \mathbf{k}_{\perp}\right)\right|^{2}=(1+\tau)(1-x)^{\tau},
$$

which is normalized to 1

$$
\int_{0}^{1} d x \rho_{\tau}(x)=1
$$

and gives the required behavior of parton densities at large $x \rightarrow 1$, both in the case of the pion $(1-x)^{2}$ and the nucleon $(1-x)^{3}$ (helicity non-flip density). The helicityflip density for the nucleon will be discussed in Sec.III.
Now we calculate the form factor $F_{\tau}\left(Q^{2}\right)$ and the generalized parton distribution (GPD) $H_{\tau}\left(x, Q^{2}\right)$

$$
\begin{aligned}
F_{\tau}\left(Q^{2}\right) & =\int_{0}^{1} d x H_{\tau}\left(x, Q^{2}\right) \\
& =\int_{0}^{1} d x \int \frac{d^{2} \mathbf{k}_{\perp}}{16 \pi^{3}} \psi_{\tau}^{*}\left(x, \mathbf{k}_{\perp}^{\prime}\right) \psi_{\tau}\left(x, \mathbf{k}_{\perp}\right) \\
& =\int_{0}^{1} d x \rho_{\tau}(x) \exp \left[-\frac{Q^{2}}{4 \kappa^{2}} \log (1 / x)(1-x)^{\alpha(\tau)}\right]
\end{aligned}
$$

where $\mathbf{k}_{\perp}^{\prime}=\mathbf{k}_{\perp}+(1-x) \mathbf{q}_{\perp}$ and $\mathbf{q}_{\perp}$ is the momentum transfer. One can see that the $Q^{2}$-dependence of the GPD generalizes the so-called modified Regge ansatz [6] for the nucleon helicity non-flip GPD

$$
\mathcal{H}\left(x, Q^{2}\right) \sim(1-x)^{3} e^{-\frac{Q^{2}}{\Lambda^{2}} \log (1 / x)(1-x)}
$$

for the specific choice $\tau=3$ to the case of hadrons with arbitrary twist-dimension $\tau$, where $\Lambda$ is the scale parameter. E.g. for the pion we get

$$
\mathcal{H}_{\pi}\left(x, Q^{2}\right) \sim(1-x)^{2} e^{-\frac{Q^{2}}{\Lambda^{2}} \log (1 / x)(1-x)^{2}} .
$$

It is easy to check that at large $Q^{2}$ the hadronic form factors are consistent with quark counting rules

$$
F_{\tau}\left(Q^{2}\right) \sim \frac{1}{\left(Q^{2}\right)^{\tau-1}}
$$

Next, following the ideas of Refs. [8 11, 13], we discuss the inclusion of quark masses in the proposed LFWF effective wave functions. We use a two-body approximation where $m_{1}$ and $m_{2}$ are treated as the masses of the struck quark and spectator compound, which is the spectator quark in the case of mesons, the diquark in the case of baryons, and a multiquark cluster in the case of other hadrons. Inclusion of quark masses leads to

$$
\begin{aligned}
\psi_{\tau}\left(x, \mathbf{k}_{\perp}\right) & \rightarrow \psi_{\tau}\left(x, \mathbf{k}_{\perp}, m_{1}, m_{2}\right) \\
& =N_{\tau} \sqrt{\log (1 / x)} x^{\beta_{1}}(1-x)^{\beta_{2}+\frac{\tau}{2}+\frac{\alpha(\tau)}{2}-1} \\
& \times \exp \left[-\frac{\mathcal{M}^{2}}{2 \kappa^{2}} \frac{x \log (1 / x)}{(1-x)^{1-\alpha(\tau)}}\right]
\end{aligned}
$$

where

$$
\mathcal{M}^{2}=\mathcal{M}_{0}^{2}+\frac{\mathbf{k}_{\perp}^{2}}{x(1-x)}=\frac{\mathbf{k}_{\perp}^{2}+m_{1}^{2}}{x}+\frac{\mathbf{k}_{\perp}^{2}+m_{2}^{2}}{1-x}
$$

is the invariant mass. Here $\beta_{1}$ and $\beta_{2}$ are parameters, which depend on the masses $m_{1}$ and $m_{2}$, respectively. One of these parameters (the parameter $\beta_{2}$ ) must obey the constraint $\beta_{2} \rightarrow 0$ in the chiral limit $m_{1}, m_{2} \rightarrow 0$ in order to have consistency with scaling rules. 


\section{LIGHT-FRONT QUARK-DIQUARK MODEL FOR THE NUCLEON}

In this section we consider the application of the phenomenological LFWF to the derivation of a light-front quark-diquark model for the nucleon. First we collect the well-known relations [14] between the set of nucleon Dirac and Pauli form factors $F_{1,2}^{N}(N=p, n)$ to the ones defining the distribution of valence quarks in nucleons $F_{1,2}^{q}(q=u, d)$ and the valence quark generalized parton distributions (GPDs) [15] ( $\mathcal{H}^{q}$ and $\left.\mathcal{E}^{q}\right)$

$$
\begin{aligned}
F_{i}^{p(n)}\left(Q^{2}\right) & =\frac{2}{3} F_{i}^{u(d)}\left(Q^{2}\right)-\frac{1}{3} F_{i}^{d(u)}\left(Q^{2}\right) \\
F_{1}^{q}\left(Q^{2}\right) & =\int_{0}^{1} d x \mathcal{H}^{q}\left(x, Q^{2}\right) \\
F_{2}^{q}\left(Q^{2}\right) & =\int_{0}^{1} d x \mathcal{E}^{q}\left(x, Q^{2}\right) .
\end{aligned}
$$

At $Q^{2}=0$ the GPDs are related to the quark densities - valence $q_{v}(x)$ and magnetic $\mathcal{E}_{q}(x)$

$$
\mathcal{H}^{q}(x, 0)=q_{v}(x), \quad \mathcal{E}^{q}(x, 0)=\mathcal{E}^{q}(x),
$$

which are normalized as

$$
\begin{aligned}
& n_{q}=F_{1}^{q}(0)=\int_{0}^{1} d x q_{v}(x), \\
& \kappa_{q}=F_{2}^{q}(0)=\int_{0}^{1} d x \mathcal{E}^{q}(x) .
\end{aligned}
$$

The number of $u$ or $d$ valence quarks in the proton is denoted by $n_{q}$ and $\kappa_{q}$ is the quark anomalous magnetic moment.

Next we recall the definitions of the nucleon Sachs form factors $G_{E / M}\left(Q^{2}\right)$ and the electromagnetic $\left\langle r_{E / M}^{2}\right\rangle^{N}$ radii in terms of the Dirac and Pauli form factors $F_{1,2}^{N}\left(Q^{2}\right)$

$$
\begin{aligned}
G_{E}^{N}\left(Q^{2}\right) & =F_{1}^{N}\left(Q^{2}\right)-\frac{Q^{2}}{4 m_{N}^{2}} F_{2}^{N}\left(Q^{2}\right), \\
G_{M}^{N}\left(Q^{2}\right) & =F_{1}^{N}\left(Q^{2}\right)+F_{2}^{N}\left(Q^{2}\right), \\
\left\langle r_{E}^{2}\right\rangle^{N} & =-\left.6 \frac{d G_{N}^{E}\left(Q^{2}\right)}{d Q^{2}}\right|_{Q^{2}=0}, \\
\left\langle r_{M}^{2}\right\rangle^{N} & =-\left.\frac{6}{G_{M}^{N}(0)} \frac{d G_{M}^{N}\left(Q^{2}\right)}{d Q^{2}}\right|_{Q^{2}=0},
\end{aligned}
$$

where $G_{M}^{N}(0) \equiv \mu_{N}$ is the nucleon magnetic moment.

The light-front representation [16, 17] for the Dirac and
Pauli quark form factors is

$$
\begin{aligned}
F_{1}^{q}\left(Q^{2}\right) & =\int_{0}^{1} d x \int \frac{d^{2} \mathbf{k}_{\perp}}{16 \pi^{3}}\left[\psi_{+q}^{+*}\left(x, \mathbf{k}_{\perp}^{\prime}\right) \psi_{+q}^{+}\left(x, \mathbf{k}_{\perp}\right)\right. \\
& \left.+\psi_{-q}^{+*}\left(x, \mathbf{k}_{\perp}^{\prime}\right) \psi_{-q}^{+}\left(x, \mathbf{k}_{\perp}\right)\right] \\
F_{2}^{q}\left(Q^{2}\right) & =-\frac{2 M_{N}}{q^{1}-i q^{2}} \int_{0}^{1} d x \int \frac{d^{2} \mathbf{k}_{\perp}}{16 \pi^{3}} \\
& \times\left[\psi_{+q}^{+*}\left(x, \mathbf{k}_{\perp}^{\prime}\right) \psi_{+q}^{-}\left(x, \mathbf{k}_{\perp}\right)\right. \\
& \left.+\psi_{-q}^{+*}\left(x, \mathbf{k}_{\perp}^{\prime}\right) \psi_{-q}^{-}\left(x, \mathbf{k}_{\perp}\right)\right]
\end{aligned}
$$

Here $M_{N}$ is the nucleon mass, $\psi_{\lambda_{q} q}^{\lambda_{N}}\left(x, \mathbf{k}_{\perp}\right)$ are the LFWFs with specific helicities of the nucleon $\lambda_{N}= \pm$ and the struck quark $\lambda_{q}= \pm$, where plus and minus correspond to $+\frac{1}{2}$ and $-\frac{1}{2}$, respectively. We work in the frame with $q=\left(0,0, \mathbf{q}_{\perp}\right)$, and therefore the Euclidean momentum squared is $Q^{2}=\mathbf{q}_{\perp}^{2}$.

In the quark-scalar diquark model the generic ansatz for the LFWFs reads

$$
\begin{aligned}
\psi_{+q}^{+}\left(x, \mathbf{k}_{\perp}\right) & =\frac{m_{q}+x M_{N}}{x} \varphi_{q}\left(x, \mathbf{k}_{\perp}\right), \\
\psi_{-q}^{+}\left(x, \mathbf{k}_{\perp}\right) & =-\frac{k^{1}+i k^{2}}{x}(1-x) \mu_{q} \varphi_{q}\left(x, \mathbf{k}_{\perp}\right), \\
\psi_{+q}^{-}\left(x, \mathbf{k}_{\perp}\right) & =\frac{k^{1}-i k^{2}}{x}(1-x) \mu_{q} \varphi_{q}\left(x, \mathbf{k}_{\perp}\right), \\
\psi_{-q}^{-}\left(x, \mathbf{k}_{\perp}\right) & =\frac{m_{q}+x M_{N}}{x} \varphi_{q}\left(x, \mathbf{k}_{\perp}\right)
\end{aligned}
$$

where $m_{q}=m_{1 q}$ is the mass of struck quark; $\varphi_{q}\left(x, \mathbf{k}_{\perp}\right)$ is the wave function, which is related to the proposed LFWF (13) as

$$
\begin{aligned}
\varphi_{q}\left(x, \mathbf{k}_{\perp}\right) & =N_{q} \sqrt{\log (1 / x)} x^{\beta_{1 q}}(1-x)^{\beta_{2 q}+1} \\
& \times \exp \left[-\frac{\mathcal{M}^{2}}{2 \kappa^{2}} x \log (1 / x)\right] .
\end{aligned}
$$

Here we include the flavor dependence effects in the normalization constant

$$
\begin{aligned}
N_{q} & =\frac{4 \pi \sqrt{n_{q}}}{\kappa M_{N}}\left[\int_{0}^{1} d x x^{2 \beta_{1 q}}(1-x)^{3+2 \beta_{2 q}}\right. \\
& \left.\times R_{q}(x) e^{-\frac{\mathcal{M}_{0}^{2}}{\kappa^{2}} x \log (1 / x)}\right]^{-1 / 2}, \\
R_{q}(x) & =\left(1+\frac{m_{1 q}}{x M_{N}}\right)^{2}+\frac{\kappa^{2} \mu_{q}^{2}}{M_{N}^{2}} \frac{(1-x)^{3}}{\log (1 / x) x^{2}}
\end{aligned}
$$

and in the parameters $\beta_{1 q}$ and $\beta_{2 q}$. The parameters $\mu_{q}$ $(q=u, d)$ are fixed from the description of the nucleon 
magnetic moments. In the LFWF $\psi_{\mp q}^{ \pm}\left(x, \mathbf{k}_{\perp}\right)$ we included an extra factor $(1-x)$ in order to generate an extra power $(1-x)^{2}$ in the helicity-flip parton density $\mathcal{E}^{q}(x) \sim(1-x)^{5}$ in comparison with the helicity non-flip density $q_{v}(x) \sim(1-x)^{3}$. Notice that the extra power of $(1-x)$ in the functions $\psi_{\mp q}^{ \pm}\left(x, \mathbf{k}_{\perp}\right)$ is consistent with results of the soft-wall AdS/QCD, where the functions $\psi_{\mp q}^{ \pm}\left(x, \mathbf{k}_{\perp}\right)$ and $\psi_{ \pm q}^{ \pm}\left(x, \mathbf{k}_{\perp}\right)$ have holographic analogues - the bulk profiles of left- and right-handed AdS fermion fields with spin $\left.\frac{1}{2}[18]-21\right]$.

Substituting our ansatz for the LFWF (21) in Eq. (19) and integrating over $\mathbf{k}_{\perp}$ we get the following expressions for the quark form factors:

$$
\begin{aligned}
F_{1}^{q}\left(Q^{2}\right) & =C_{q} \int_{0}^{1} d x x^{2 \beta_{1 q}}(1-x)^{3+2 \beta_{2 q}} R_{q}\left(x, Q^{2}\right) \\
& \times \exp \left[-\frac{Q^{2}}{4 \kappa^{2}} \log (1 / x)(1-x)\right] \\
& \times \exp \left[-\frac{\mathcal{M}_{0}^{2}}{\kappa^{2}} x \log (1 / x)\right], \\
F_{2}^{q}\left(Q^{2}\right) & =C_{q} \int_{0}^{1} \frac{2 d x}{x} \mu_{q}\left(1+\frac{m_{1 q}}{x M_{N}}\right) x^{2 \beta_{1 q}}(1-x)^{5+2 \beta_{2 q}} \\
& \times \exp \left[-\frac{Q^{2}}{4 \kappa^{2}} \log (1 / x)(1-x)\right] \\
& \times \exp \left[-\frac{\mathcal{M}_{0}^{2}}{\kappa^{2}} x \log (1 / x)\right],
\end{aligned}
$$

where

$$
\begin{aligned}
C_{q} & =N_{q}^{2}\left(\frac{\kappa M_{N}}{4 \pi}\right)^{2} \\
R_{q}\left(x, Q^{2}\right) & =R_{q}(x)-\frac{Q^{2}}{4 M_{N}^{2}} \frac{(1-x)^{4}}{x^{2}} \mu_{q}^{2} .
\end{aligned}
$$

In particular, one possible set of parameters, which gives a reasonable description of nucleon electromagnetic propertie is $m_{1}=7 \mathrm{MeV}$ (mass of active quark), $m_{2}=190 \mathrm{MeV}$ (mass of spectator diquark), $\mu_{u}=0.3$, $\mu_{d}=-1.2, \beta_{1 u}=1.1, \beta_{2 u}=0.2, \beta_{1 d}=1.5, \beta_{2 d}=$ $0.26, \kappa=300 \mathrm{MeV}$ (see our results in comparison with data [22] in Table I).

Now we demonstrate that the obtained results obey the model-independent scaling laws. Considering the limit $x \rightarrow 1$ where $\log (1 / x) \sim 1-x$ and the limit $Q^{2} \rightarrow \infty$ [changing $x \rightarrow 1-x$ and then rescaling $x \rightarrow\left(2 \kappa / \sqrt{Q^{2}}\right) x$ ], we prove the DYW duality and quark scaling rules

$$
\begin{aligned}
& F_{1}^{q}\left(Q^{2}\right) \sim \frac{1}{\left(Q^{2}\right)^{2+\beta_{2 q}}}, \\
& F_{2}^{q}\left(Q^{2}\right) \sim \frac{1}{\left(Q^{2}\right)^{3+\beta_{2 q}}}, \\
& \frac{F_{2}^{q}\left(Q^{2}\right)}{F_{1}^{q}\left(Q^{2}\right)} \sim \frac{1}{Q^{2}}
\end{aligned}
$$

TABLE I: Electromagnetic properties of nucleons

\begin{tabular}{|c|c|c|}
\hline Quantity & Our results & Data [22] \\
\hline$\mu_{p}$ (in n.m.) & 2.793 & 2.793 \\
\hline$\mu_{n}$ (in n.m.) & -1.826 & -1.913 \\
\hline$r_{E}^{p}(\mathrm{fm})$ & 0.741 & $0.8768 \pm 0.0069$ \\
\hline$\left\langle r_{E}^{2}\right\rangle^{n}\left(\mathrm{fm}^{2}\right)$ & -0.116 & $-0.1161 \pm 0.0022$ \\
\hline$r_{M}^{p}(\mathrm{fm})$ & 0.817 & $0.777 \pm 0.013 \pm 0.010$ \\
\hline$r_{M}^{n}(\mathrm{fm})$ & 0.851 & $0.862_{-0.008}^{+0.009}$ \\
\hline
\end{tabular}

and

$$
q_{v}(x) \sim(1-x)^{3+2 \beta_{2 q}}, \quad \mathcal{E}^{q}(x) \sim(1-x)^{5+2 \beta_{2 q}},
$$

where $\beta_{2 q} \rightarrow 0$ in the chiral limit. Therefore, the nucleon form factors and parton densities are consistent with the DYW duality and quark counting rules. We plan to present a detailed numerical analysis of GPDs and parton densities in a forthcoming paper [23].

\section{CONCLUSION}

In conclusion we want to stress again the main result of our paper: we demonstrated how to construct a lightfront quark model consistent with the model-independent scaling laws - the DYW duality [1] and quark counting rules [3]. We plan to perform a detailed analysis of baryon properties using the light-front quark model proposed in the present letter.

\section{Acknowledgments}

The authors thank Stan Brodsky and Guy de Téramond for useful discussions. This work was supported by the DFG under Contract No. LY 114/2-1, by FONDECYT (Chile) under Grant No. 1100287 and by CONICYT (Chile) under Grant No. 7912010025. The work is done partially under the project 2.3684.2011 of Tomsk State University. V. E. L. would like to thank Departamento de Física y Centro Científico Tecnológico de Valparaíso (CCTVal), Universidad Técnica Federico Santa María, Valparaíso, Chile for warm hospitality. 
[1] S. D. Drell and T. -M. Yan, Phys. Rev. Lett. 24, 181 (1970); G. B. West, Phys. Rev. Lett. 24, 1206 (1970).

[2] E. D. Bloom and F. J. Gilman, Phys. Rev. Lett. 25, 1140 (1970).

[3] S. J. Brodsky and G. R. Farrar, Phys. Rev. Lett. 31, 1153 (1973); V. A. Matveev, R. M. Muradian and A. N. Tavkhelidze, Lett. Nuovo Cim. 7, 719 (1973).

[4] S. J. Brodsky, M. Burkardt and I. Schmidt, Nucl. Phys. B 441, 197 (1995) hep-ph/9401328.

[5] F. Yuan, Phys. Rev. D 69, 051501 (2004) hep-ph/0311288.

[6] M. Burkardt, Int. J. Mod. Phys. A 18, 173 (2003) hep-ph/0207047.

[7] M. Diehl, T. Feldmann, R. Jakob and P. Kroll, Eur. Phys. J. C 39, 1 (2005) hep-ph/0408173; M. Guidal, M. V. Polyakov, A. V. Radyushkin and M. Vanderhaeghen, Phys. Rev. D 72, 054013 (2005) hep-ph/0410251; O. V. Selyugin and O. V. Teryaev, Phys. Rev. D 79, 033003 (2009) arXiv:0901.1786 [hep-ph]].

[8] S. J. Brodsky, G. F. de Teramond, Phys. Rev. D 77, 056007 (2008) arXiv:0707.3859 [hep-ph]].

[9] S. J. Brodsky and G. F. de Teramond, arXiv:0802.0514 [hep-ph].

[10] A. Vega, I. Schmidt, T. Branz, T. Gutsche and V. E. Lyubovitskij, Phys. Rev. D 80, 055014 (2009) arXiv:0906.1220 [hep-ph]].

[11] T. Branz, T. Gutsche, V. E. Lyubovitskij, I. Schmidt, A. Vega, Phys. Rev. D 82, 074022 (2010) arXiv:1008.0268 [hep-ph]];

[12] S. J. Brodsky, F. -G. Cao and G. F. de Teramond, Phys. Rev. D 84, 075012 (2011) arXiv:1105.3999 [hep-ph]].

[13] T. Gutsche, V. E. Lyubovitskij, I. Schmidt and A. Vega, Phys. Rev. D 87, 056001 (2013) arXiv:1212.5196 [hep$\mathrm{ph}]$;
[14] A. V. Radyushkin, Phys. Rev. D 58, 114008 (1998) hep-ph/9803316.

[15] D. Mueller, D. Robaschik, B. Geyer, F. M. Dittes and J. Horejsi, Fortsch. Phys. 42, 101 (1994) arXiv:hep-ph/9812448; X. -D. Ji, Phys. Rev. Lett. 78, 610 (1997) hep-ph/9603249] A. V. Radyushkin, Phys. Rev. D 56, 5524 (1997) hep-ph/9704207).

[16] S. J. Brodsky, S. D. Drell, Phys. Rev. D 22, 2236 (1980).

[17] S. J. Brodsky and D. S. Hwang, Nucl. Phys. B 543, 239 (1999) hep-ph/9806358; S. J. Brodsky, D. S. Hwang, B. -Q. Ma and I. Schmidt, Nucl. Phys. B 593, 311 (2001) hep-th/0003082; S. J. Brodsky, M. Diehl and D. S. Hwang, Nucl. Phys. B 596, 99 (2001) hep-ph/0009254.

[18] Z. Abidin and C. E. Carlson, Phys. Rev. D 79, 115003 (2009) arXiv:0903.4818 [hep-ph]].

[19] A. Vega, I. Schmidt, T. Gutsche and V. E. Lyubovitskij, Phys. Rev. D 83, 036001 (2011) arXiv:1010.2815 [hep$\mathrm{ph}]$.

[20] G. F. de Teramond and S. J. Brodsky, AIP Conf. Proc. 1432, 168 (2012) arXiv:1108.0965 [hep-ph]]; S. J. Brodsky and G. F. de Teramond, AIP Conf. Proc. 1388, 22 (2011) arXiv:1103.1186 [hep-ph]].

[21] T. Gutsche, V. E. Lyubovitskij, I. Schmidt and A. Vega, Phys. Rev. D 85, 076003 (2012) arXiv:1108.0346 [hepph]]; Phys. Rev. D 86, 036007 (2012) arXiv:1204.6612 [hep-ph]]; Phys. Rev. D 87, 016017 (2013) arXiv:1212.6252 [hep-ph]].

[22] J. Beringer et al. [Particle Data Group Collaboration], Phys. Rev. D 86, 010001 (2012).

[23] A. Vega, I. Schmidt, T. Gutsche, V. E. Lyubovitskij, in preparation. 